\title{
Injective stability for odd-dimensional unitary $K_{1}$
}

\author{
Weibo Yu \\ Communicated by John S. Wilson
}

\begin{abstract}
In this paper, under the usual stable range condition, a decomposition theorem for the elementary subgroup is obtained, and the injective stability theorem for odddimensional unitary $K_{1}$ is proved.
\end{abstract}

\section{Introduction}

Stability results for classical $K_{1}$, beginning with Bass [5] and Vaserstein [10] for linear $K_{1}$, Bak and Tang [2] for Hermitian $K_{1}$, Bak, Petrov and Tang [3] and Tang [9] for quadratic $K_{1}$, and the author [11] for odd unitary $K_{1}$, have been available in the literature for a long time and play an important role in the theory of classical groups. The stability theorems in $[2,3,9,11]$ are all proved under the $\Lambda$-stable range condition, which is weaker than its predecessors. The difficulty in the proof of stability results is injective stability, which is always concerned with a decomposition of the elementary subgroup. Does injective stability hold under the usual stable range condition which is weaker than the $\Lambda$-stable range condition? In [8], Sinchuk proved injective stability for unitary $K_{1}$ under the usual stable range condition. In [1], Ambily proved injective stability for $K_{1}$ of Roy's group under the usual stable range condition over a commutative ring.

Recently, Bak and Preusser [4] introduced odd-dimensional unitary groups, which are isomorphic to Petrov's odd hyperbolic unitary groups [7] such that $V_{0}=R$ and contain as special cases the groups $\operatorname{GL}_{2 n+1}(R)$, where $R$ is any ring, the classical groups $O_{2 n+1}(R)$ and $\operatorname{Sp}_{2 n+1}(R)$, where $R$ is any commutative ring, and further all even-dimensional unitary groups $U_{2 n}(R, \Lambda)$, where $(R, \Lambda)$ is any form ring (see [4, Example 15]). In this paper, using the usual stable range condition, we prove the injective stability theorem for odd-dimensional unitary $K_{1}$, which generalizes similar results given in previous papers.

Let $R$ be an associative ring with identity and $(R, \Delta)$ a Hermitian form ring. Denote by $U_{2 n+1}(R, \Delta)$ the odd-dimensional unitary group and by $E U_{2 n+1}(R, \Delta)$ mental Research Funds for the Central Universities, CHD (No. 300102129106). 
its elementary subgroup. Under certain assumptions on $R, E U_{2 n+1}(R, \Delta)$ is normal in $U_{2 n+1}(R, \Delta)$ (see [4, Theorem 39], [7, Theorem 4], [11, Corollary 3.3], [12, Theorem 4.4]). Set

$$
K U_{1, n}(R, \Delta):=U_{2 n+1}(R, \Delta) / E U_{2 n+1}(R, \Delta) .
$$

In this paper, we consider this object as a coset space. Under the embedding

$$
\begin{aligned}
\phi_{2 n+1}^{2 n+3}: U_{2 n+1}(R, \Delta) & \rightarrow U_{2 n+3}(R, \Delta), \\
\sigma & \mapsto\left(\begin{array}{lll}
1 & 0 & 0 \\
0 & \sigma & 0 \\
0 & 0 & 1
\end{array}\right),
\end{aligned}
$$

we have the stabilization map $K U_{1, n}(R, \Delta) \rightarrow K U_{1, n+1}(R, \Delta)$. Then we can get the following injective stability theorem.

Theorem 1.1. Let $n \geq \operatorname{sr}(R)+2$. Then the map

$$
K U_{1, n-1}(R, \Delta) \rightarrow K U_{1, n}(R, \Delta)
$$

is injective.

The rest of the paper is organized as follows. In Section 2, we recall the necessary notation. In Section 3, we recall in detail the definitions of the odd-dimensional unitary group $U_{2 n+1}(R, \Delta)$, elementary subgroup $E U_{2 n+1}(R, \Delta)$, and the usual stable range condition. In Section 4 , we establish a decomposition theorem for $E U_{2 n+1}(R, \Delta)$ and prove our main result Theorem 1.1.

\section{Notation}

In this paper, denote by $\mathbb{N}$ the set of all positive integers. If $m, n \in \mathbb{N}$, the set of all $m \times n$ matrices with entries in the ring $R$ is denoted by $\mathrm{M}_{m \times n}(R)$. Let $A_{i j}$ denote the entry at position $(i, j)$ if $A \in \mathrm{M}_{m \times n}(R)$. Denote by $A^{t}$ the transpose of $A$, that is, $\left(A^{t}\right)_{i j}=A_{j i}$. Denote the $i$-th row of $A$ by $A_{i *}$ and the $j$-th column of $A$ by $A_{* j}$. Set $\mathrm{M}_{n}(R):=\mathrm{M}_{n \times n}(R)$. The identity matrix in $\mathrm{M}_{n}(R)$ is denoted by $e$ or $E_{n}$. If $A \in \mathrm{M}_{n}(R)$ is invertible, the entry of $A^{-1}$ at position $(i, j)$ is denoted by $A_{i j}^{\prime}$. Further, we denote by $R^{n}$ the set of all columns $\left(a_{1}, \ldots, a_{n}\right)^{t}$ with entries in $R$.

Let $G$ be a group and $H$ a subset of $G$. Denote by $\langle H\rangle$ the subgroup of $G$ generated by $H$. If $x, y \in G$, let $x^{y}:=y^{-1} x y$ and $[x, y]:=x y x^{-1} y^{-1}$. Set

$$
H^{x}:=\left\langle\left\{h^{x} \mid h \in H\right\}\right\rangle \text {. }
$$




\section{Odd-dimensional unitary groups}

\subsection{Hermitian form ring}

Our concept closely follows [4]. Let $R$ be an associative ring with identity 1 . Recall that an anti-isomorphism ${ }^{-}: R \rightarrow R, a \mapsto \bar{a}$ on $R$ is a bijective map satisfying $\overline{a+b}=\bar{a}+\bar{b}, \overline{a b}=\bar{b} \bar{a}$ for all $a, b \in R$ and $\overline{1}=1$. Let $\lambda \in R$ with the property that $\overline{\bar{a}}=\lambda a \bar{\lambda}$ for all $a \in R$. It is easy to see that $\bar{\lambda}=\lambda^{-1}$. The element $\lambda$ is called a symmetry for ${ }^{-}$and the triple $\left(R,{ }^{-}, \lambda\right)$ a ring with involution and symmetry. A Hermitian ring is a quadruple $\left(R,{ }^{-}, \lambda, \mu\right)$, where $\left(R,{ }^{-}, \lambda\right)$ is a ring with involution and symmetry and $\mu \in R$ such that $\mu=\bar{\mu} \lambda$.

Remark 3.1. (1) Define the map : $R \rightarrow R, a \mapsto \underline{a}:=\bar{\lambda} \bar{a} \lambda$. It is the inverse map of $^{-}$. One checks easily that $(R, \ldots, \underline{\lambda}, \mu)$ is a Hermitian ring.

(2) For any $n \in \mathbb{N},\left(\mathrm{M}_{n}(R),{ }^{*}, \lambda e, \bar{\mu} e\right)$ is a Hermitian ring, where

$$
A^{*}:=\bar{A}^{t}=\left(\left(\bar{A}_{i j}\right)_{i j}\right)^{t}=\left(\bar{A}_{j i}\right)_{i j} \quad \text { for any } A \in \mathrm{M}_{n}(R) .
$$

Let $R^{\bullet}$ be the underlying set of the ring equipped with the multiplication of the ring, but not the addition of the ring. A (right) $R^{\bullet}$-module is a not necessarily abelian group $(G, \dot{+})$ equipped with a map $\circ: G \times R^{\bullet} \rightarrow G,(x, a) \mapsto x \circ a$, satisfying

(1) $x \circ 0=0$ for all $x \in G$,

(2) $x \circ 1=x$ for all $x \in G$,

(3) $(x \circ a) \circ b=x \circ(a b)$ for all $x \in G$ and $a, b \in R$,

(4) $(x \dot{+} y) \circ a=(x \circ a) \dot{+}(y \circ a)$ for all $x, y \in G$ and $a \in R$.

We treat $\circ$ as an operator with higher priority than $\dot{+}$. An $R$-module is canonically an $R^{\bullet}$-module, but not conversely. Let $G$ and $G^{\prime}$ be $R^{\bullet}$-modules. By a homomorphism of $R^{\bullet}$-modules, one means a group homomorphism $f: G \rightarrow G^{\prime}$ such that $f(x \circ a)=f(x) \circ a$ for all $x \in G$ and $a \in R$. By an $R^{\bullet}$-submodule $N$, we mean a subgroup $N$ of $G$ which is o-stable, that is, $x \circ a \in N$ for all $x \in N$ and $a \in R$.

Let $\left(R,{ }^{-}, \lambda, \mu\right)$ be a Hermitian ring. Consider the Heisenberg group $\mathfrak{Y}$, where $\mathfrak{Y}=R \times R$ as a set, with a composition operation “+ + ", satisfying

$$
\left(a_{1}, b_{1}\right) \dot{+}\left(a_{2}, b_{2}\right)=\left(a_{1}+a_{2}, b_{1}+b_{2}-\bar{a}_{1} \mu a_{2}\right) .
$$

It is easy to see that the law $\dot{+}$ is associative with identity $(0,0)$, and the inverse is given by

$$
\dot{-}(a, b)=(-a,-b-\bar{a} \mu a) \quad \text { for any }(a, b) \in \mathfrak{Y} \text {. }
$$


Furthermore, supply $\bigvee$ with a right action of $R^{\bullet}$ through $(a, b) \circ c=(a c, \bar{c} b c)$. $\eta$ becomes an $R^{\bullet}$-module.

Let $(R,+)$ have the $R^{\bullet}$-module structure defined by $a \circ b=\bar{b} a b$. Define the trace map tr: $\mathfrak{Y} \rightarrow R$ by $\operatorname{tr}((a, b))=\bar{a} \mu a+b+\bar{b} \lambda$. It is easy to see that $\operatorname{tr}$ is a homomorphism of $R^{\bullet}$-modules.

Set

$$
\Delta_{\min }:=\{(0, a-\bar{a} \lambda) \mid a \in R\} \quad \text { and } \quad \Delta_{\max }:=\operatorname{ker}(\operatorname{tr}) .
$$

An odd form parameter of $\left(R,{ }^{-}, \lambda, \mu\right)$ is an $R^{\bullet}$-submodule $\Delta$ of $\mathfrak{Y}$ such that $\Delta_{\min } \leq \Delta \leq \Delta_{\max }$. The pair $\left(\left(R,{ }^{-}, \lambda, \mu\right), \Delta\right)$ is called a Hermitian form ring and is abbreviated by $(R, \Delta)$.

\subsection{Odd-dimensional unitary groups}

Let $(R, \Delta)$ be a Hermitian form ring and $n \in \mathbb{N}$. Consider the right $R$-module $M:=R^{2 n+1}$ with the fixed basis $\left(e_{1}, \ldots, e_{n}, e_{0}, e_{-n}, \ldots, e_{-1}\right)$, where $e_{i}$ is the column vector whose $i$-th entry $(1 \leq i \leq-1)$ is 1 and whose other entries are 0 . If $u \in M$, we call $\left(u_{1}, \ldots, u_{n}, u_{-n}, \ldots, u_{-1}\right)^{t} \in R^{2 n}$ the hyperbolic part of $u$ and denote it by $u_{\mathrm{hb}}$. Define the maps

$$
\begin{aligned}
h: M \times M & \rightarrow R, \\
(u, v) & \mapsto u^{*}\left(\begin{array}{ccc}
0 & 0 & p \\
0 & \mu & 0 \\
p \lambda & 0 & 0
\end{array}\right) v=\sum_{i=1}^{n} \bar{u}_{i} v_{-i}+\bar{u}_{0} \mu v_{0}+\sum_{i=-n}^{-1} \bar{u}_{i} \lambda v_{-i},
\end{aligned}
$$

and

$q: M \rightarrow \mathfrak{Y}$,

$$
u \mapsto\left(q_{1}(u), q_{2}(u)\right):=\left(u_{0}, u_{\mathrm{hb}}^{*}\left(\begin{array}{ll}
0 & p \\
0 & 0
\end{array}\right) u_{\mathrm{hb}}\right)=\left(u_{0}, \sum_{i=1}^{n} \bar{u}_{i} u_{-i}\right),
$$

where $u^{*}=\bar{u}^{t}, u_{\mathrm{hb}}^{*}=\bar{u}_{\mathrm{hb}}^{t}$ and $p \in \mathrm{M}_{n}(R)$ is the matrix with 1 on the skew diagonal and 0 elsewhere. One checks easily the following lemma.

Lemma 3.2. The following statements hold.

(1) $h$ is a $\lambda$-Hermitian form, that is,

(a) $h$ is biadditive,

(b) $h(u a, v b)=\bar{a} h(u, v) b$ for all $u, v \in M$ and $a, b \in R$,

(c) $h(u, v)=\overline{h(v, u)} \lambda$ for all $u, v \in M$. 
(2) q has the following properties:

(a) $q(u a)=q(u) \circ a$ for all $u \in M$ and $a \in R$,

(b) $q(u+v) \equiv q(u) \dot{+} q(v) \dot{+}(0, h(u, v)) \bmod \Delta_{\min }$ for all $u, v \in M$,

(c) $\operatorname{tr}(q(u))=h(u, u)$ for all $u \in M$.

The odd-dimensional unitary group $U_{2 n+1}(R, \Delta)$ is the group consisting of all elements $\sigma \in \mathrm{GL}_{2 n+1}(R)$ satisfying

$$
\begin{aligned}
h(\sigma u, \sigma v) & =h(u, v), \\
q(\sigma u) & \equiv q(u) \bmod \Delta
\end{aligned}
$$

for all $u, v \in M$. These groups are isomorphic to Petrov's odd hyperbolic unitary groups such that $V_{0}=R$ and contain as special cases the groups $\operatorname{GL}_{2 n+1}(R)$, where $R$ is any ring, the classical groups $O_{2 n+1}(R)$ and $\operatorname{Sp}_{2 n+1}(R)$, where $R$ is any commutative ring, and further all even-dimensional unitary groups $U_{2 n}(R, \Lambda)$, where $(R, \Lambda)$ is any form ring.

Set

$$
\Theta:=\{1, \ldots, n, 0,-n, \ldots,-1\}, \quad \Theta_{\mathrm{hb}}:=\Theta \backslash\{0\} .
$$

Let $\epsilon(i)$ be 1 for $i=1, \ldots, n$ and -1 for $i=-n, \ldots,-1$.

Lemma 3.3. Let $\sigma \in \mathrm{GL}_{2 n+1}(R)$. Then $\sigma \in U_{2 n+1}(R, \Delta)$ if and only if conditions (1) and (2) below hold.

(1) We have

$$
\begin{aligned}
\sigma_{i j}^{\prime} & =\lambda^{-(\epsilon(i)+1) / 2} \bar{\sigma}_{-j,-i} \lambda^{(\epsilon(j)+1) / 2} & & \text { for all } i, j \in \Theta_{\mathrm{hb}}, \\
\mu \sigma_{0 j}^{\prime} & =\bar{\sigma}_{-j, 0} \lambda^{(\epsilon(j)+1) / 2} & & \text { for all } j \in \Theta_{\mathrm{hb}}, \\
\sigma_{i 0}^{\prime} & =\lambda^{-(\epsilon(i)+1) / 2} \bar{\sigma}_{0,-i} \mu & & \text { for all } i \in \Theta_{\mathrm{hb}}, \\
\mu \sigma_{00}^{\prime} & =\bar{\sigma}_{00} \mu . & &
\end{aligned}
$$

(2) $q\left(\sigma_{* j}\right) \equiv\left(\delta_{0 j}, 0\right) \bmod \Delta$ for all $j \in \Theta$.

Proof. See [4, Lemma 17].

Define the embedding $\Phi: \operatorname{GL}_{n}(R) \rightarrow U_{2 n+1}(R, \Delta)$ by

$$
\Phi(\sigma)=\left(\begin{array}{ccc}
\sigma & 0 & 0 \\
0 & 1 & 0 \\
0 & 0 & p\left(\sigma^{*}\right)^{-1} p
\end{array}\right)
$$




\subsection{The elementary subgroup}

Let $\left(R,^{-}, \lambda, \mu\right)$ be a Hermitian ring. We defined an $R^{\bullet}$-module structure on $\mathfrak{Y}$. Let $(R, \underline{\lambda}, \underline{\mu})$ be the Hermitian ring defined in Remark $3.1(1)$, and let $\mathfrak{Y}$ be the Heisenberg group corresponding to $(R,-\underline{\lambda}, \mu)$. Since the underlying set of both $\left(R,{ }^{-}, \lambda, \mu\right)$ and $(R,-, \underline{\lambda}, \mu)$ is $R \times R$, we get another $R^{\bullet}$-module structure on $R \times R$ if we replace the Hermitian ring $\left(R,{ }^{-}, \lambda, \mu\right)$ by $(R,-\underline{\lambda}, \underline{\mu})$. We denote the group operation (resp. scalar multiplication) defined by $\left(R,{ }^{-}, \lambda, \mu\right)$ and $(R,, \underline{\lambda}, \mu)$ on $R \times R$ by $\dot{+}_{1}$ and $\dot{+}_{-1}$ (resp. $\circ_{1}$ and $\circ_{-1}$ ), respectively. Set

$$
\Delta^{1}:=\Delta \quad \text { and } \quad \Delta^{-1}:=\{(a, b) \in R \times R \mid(a, \bar{b}) \in \Delta\} .
$$

It is easy to see that $\left((R,-, \underline{\lambda}, \mu), \Delta^{-1}\right)$ is a Hermitian form ring.

If $i, j \in \Theta$, let $e^{i j}$ denote the matrix in $\mathrm{M}_{2 n+1}(R)$ with 1 in the $(i, j)$-th position and 0 elsewhere. If $i, j \in \Theta_{\mathrm{hb}}, i \neq \pm j$ and $a \in R$, the element

$$
T_{i j}(a):=e+a e^{i j}-\lambda^{(\epsilon(j)-1) / 2} \bar{a} \lambda^{(1-\epsilon(i)) / 2} e^{-j,-i} \quad \text { of } U_{2 n+1}(R, \Delta)
$$

is called an (elementary) short root matrix. If $i \in \Theta_{\mathrm{hb}}$ and $(a, b) \in \Delta^{-\epsilon(i)}$, the element

$$
T_{i}(a, b):=e+a e^{0,-i}-\lambda^{-(1+\epsilon(i)) / 2} \bar{a} \mu e^{i 0}+b e^{i,-i} \text { of } U_{2 n+1}(R, \Delta)
$$

is called an (elementary) extra short root matrix. The extra short root matrices of the kind $T_{i}(0, b)=e+b e^{i,-i}$ are called (elementary) long root matrices. An element of $U_{2 n+1}(R, \Delta)$ is called elementary matrix if it is a short or extra short root matrix. The elementary subgroup $E U_{2 n+1}(R, \Delta)$ is the subgroup of $U_{2 n+1}(R, \Delta)$ generated by all elementary matrices. The coset space

$$
K U_{1, n}(R, \Delta):=U_{2 n+1}(R, \Delta) / E U_{2 n+1}(R, \Delta)
$$

is called the odd-dimensional unitary $K_{1}$-functor. Under the embedding

$$
\begin{aligned}
\phi_{2 n+1}^{2 n+3}: U_{2 n+1}(R, \Delta) & \rightarrow U_{2 n+3}(R, \Delta), \\
\sigma & \mapsto\left(\begin{array}{lll}
1 & 0 & 0 \\
0 & \sigma & 0 \\
0 & 0 & 1
\end{array}\right),
\end{aligned}
$$

it is easy to see that $\phi_{2 n+1}^{2 n+3}\left(E U_{2 n+1}(R, \Delta)\right) \subseteq E U_{2 n+3}(R, \Delta)$. Therefore, we have the stabilization map $K U_{1, n}(R, \Delta) \rightarrow K U_{1, n+1}(R, \Delta)$. 
The elementary matrices satisfy the following properties:

$$
\begin{aligned}
& T_{i j}(a)=T_{-j,-i}\left(-\lambda^{(\epsilon(j)-1) / 2} \bar{a} \lambda^{(1-\epsilon(i)) / 2}\right), \\
& T_{i j}(a) T_{i j}(b)=T_{i j}(a+b), \\
& {\left[T_{i j}(a), T_{k l}(b)\right] }=e \quad \text { if } k \neq-i, j \text { and } l \neq i,-j, \\
& {\left[T_{i j}(a), T_{j k}(b)\right] }=T_{i k}(a b) \quad \text { if } k \neq \pm i, \\
& {\left[T_{i j}(a), T_{j,-i}(b)\right] }=T_{i}\left(0, a b-\lambda^{-(1+\epsilon(i)) / 2} \bar{b} \bar{a} \lambda^{(1-\epsilon(i)) / 2}\right), \\
& T_{i}\left(a_{1}, b_{1}\right) T_{i}\left(a_{2}, b_{2}\right)=T_{i}\left(\left(a_{1}, b_{1}\right) \dot{+}_{-\epsilon(i)}\left(a_{2}, b_{2}\right)\right), \\
& {\left[T_{i}\left(a_{1}, b_{1}\right), T_{j}\left(a_{2}, b_{2}\right)\right] }=T_{i,-j}\left(-\lambda^{-(1+\epsilon(i)) / 2} \bar{a}_{1} \mu a_{2}\right) \quad \text { if } j \neq \pm i, \\
& {\left[T_{i}\left(a_{1}, b_{1}\right), T_{i}\left(a_{2}, b_{2}\right)\right] }=T_{i}\left(0,-\lambda^{-(1+\epsilon(i)) / 2}\left(\bar{a}_{1} \mu a_{2}-\bar{a}_{2} \mu a_{1}\right)\right), \\
& {\left[T_{i j}(a), T_{k}(b, c)\right] }=e \quad \text { if } k \neq j,-i, \\
& {\left[T_{i j}(a), T_{j}(b, c)\right] }=T_{j,-i}\left(c \lambda^{(\epsilon(j)-1) / 2} \bar{a} \lambda^{(1-\epsilon(i)) / 2}\right) \\
& \cdot T_{i}\left(b \lambda^{(\epsilon(j)-1) / 2} \bar{a} \lambda^{(1-\epsilon(i)) / 2},\right. \\
&\left.a c \lambda^{(\epsilon(j)-1) / 2} \bar{a} \lambda^{(1-\epsilon(i)) / 2}\right) .
\end{aligned}
$$

Let $i, j \in \Theta_{\mathrm{hb}}$ such that $i \neq \pm j$. In the following, the usual ordering of the numbers $-n, \ldots,-1,0,1, \ldots, n$ is used. Denote by $T_{i j}$ the subgroup consisting of all $T_{i j}(a)$ with $a \in R$ and by $T_{i}$ the subgroup consisting of all $T_{i}(a, b)$ with $(a, b) \in \Delta^{-\epsilon(i)}$. Let $n \geq 3$. We define the following subgroups of $E U_{2 n+1}(R, \Delta)$ which will be used to establish a decomposition theorem in next section.

$$
\begin{aligned}
U_{n} & =\left\langle T_{i j}, T_{i} \mid j<0<i\right\rangle, \\
U_{n}^{-} & =\left\langle T_{i j}, T_{i} \mid i<0<j\right\rangle, \\
L_{n} & =\left\langle T_{i j} \mid i, j>0\right\rangle, \\
F_{n} & =\left\langle T_{i j}, T_{i} \mid i \neq-1, j \neq 1\right\rangle, \\
Y^{+} & =\left\langle T_{n}, T_{n-1}, T_{n-1,-n}\right\rangle \leq U_{n}, \\
Y^{-} & =\left\langle T_{-n}, T_{-(n-1)}, T_{-(n-1), n}\right\rangle \leq U_{n}^{-}, \\
V^{+} & =\left\langle T_{i, n}, T_{i, n-1} \mid i=1, \ldots, n-2\right\rangle \leq L_{n}, \\
V^{-} & =\left\langle T_{n, i}, T_{n-1, i} \mid i=1, \ldots, n-2\right\rangle \leq L_{n} .
\end{aligned}
$$

Denote by $\rtimes$ the semidirect product. Set

$$
U^{+}=U_{n} \rtimes V^{+}, \quad U^{-}=U_{n}^{-} \rtimes V^{-}, \quad G_{n}=U_{n} \rtimes L_{n} .
$$




\subsection{Stable range condition}

Let $R$ be an associative ring with 1 . A vector $\left(a_{1}, \ldots, a_{n}\right)^{t}$ with $a_{i} \in R$ is called (left) unimodular if there exist elements $b_{1}, \ldots, b_{n} \in R$ such that

$$
b_{1} a_{1}+\cdots+b_{n} a_{n}=1 .
$$

The stable range condition $S_{n}$ says that, for every unimodular vector

$$
\left(a_{1}, \ldots, a_{n+1}\right)^{t} \in R^{n+1},
$$

there exist elements $b_{1}, \ldots, b_{n} \in R$ such that

$$
\left(a_{1}+b_{1} a_{n+1}, \ldots, a_{n}+b_{n} a_{n+1}\right)^{t} \in R^{n}
$$

is unimodular. The stable rank of $R$, denoted by $\operatorname{sr}(R)$, is the smallest number $n$ such that $S_{n}$ holds.

Lemma 3.4 ([8, Lemma 9]). Let $m<n$ and $\operatorname{sr}(R) \leq n-m$. Let $v$ be a unimodular column of height $n$. Then there exists a matrix $B \in \mathrm{M}_{(n-m) \times m}(R)$ such that $\left(E_{n-m}, B\right) v \in R^{n-m}$ is unimodular.

\section{Injective stability}

In this section, we get a decomposition theorem for $E U_{2 n+1}(R, \Delta)$ and prove an injective stability theorem for odd-dimensional unitary $K_{1}$ under stable range condition.

Lemma 4.1. Let $n \geq 3$. Then $E U_{2 n+1}(R, \Delta)$ is generated by $G_{n}$ and $Y^{-}$.

Proof. Denote by $H$ the subgroup of $E U_{2 n+1}(R, \Delta)$ generated by $G_{n}$ and $Y^{-}$. We only need to prove that $U_{n}^{-} \subseteq H$. Let $i=1, \ldots, n-1, j=1, \ldots, n-2$, $a \in R$ and $(b, c) \in \Delta$. It follows from (S1), (S4) and (SE2) that

$$
\begin{aligned}
T_{-n, j}(a) & =\left[T_{-n, n-1}(a), T_{n-1, j}(1)\right] \in H, \\
T_{-j, i}(a) & =\left[T_{-j,-n}(1), T_{-n, i}(a)\right] \in H, \\
T_{-j}(b, c) & =T_{-n, j}^{-1}(c)\left[T_{n j}(-1), T_{-n}(b, c)\right] \in H .
\end{aligned}
$$

Hence $H$ contains all the generators of $E U_{2 n+1}(R, \Delta)$.

Let $H_{1}, \ldots, H_{s}$ be arbitrary subsets of a group $G$. Define the Minkowski product $H_{1} \ldots H_{s}:=\left\{h_{1} \ldots h_{s} \mid h_{i} \in H_{i}, i=1, \ldots, s\right\}$. 
Lemma 4.2. Let $n \geq 3$. We have the following inclusions:

(i) $Y^{-} U^{+} \subseteq U^{+} Y^{-} Y^{+}$,

(ii) $Y^{+} U^{-} \subseteq U^{-} Y^{+} Y^{-}$,

(iii) $Y^{-} U^{+} U^{-} \subseteq U^{+} U^{-} Y^{+} Y^{-}$.

Proof. (i) Let $A=T_{n} \cup T_{n-1} \cup T_{n-1,-n}$ and $B$ be the union of all $T_{i j}$ and $T_{i}$, where $i \in\{1, \ldots, n-2\}$ and either $j<0$ or $j \in\{n-1, n\}$. Clearly, we have $U^{+}=\langle A \cup B\rangle$ and $Y^{+}=\langle A\rangle$. If $a \in A$ and $b \in B$, then $a b=[a, b] b a$.

Case 1: $a \in T_{n}$. If $b \in T_{i j}$, it follows from (SE1) that $[a, b]=e$. If $b \in T_{i}$, it follows from (E2) that $[a, b] \in T_{i,-n}$.

Case 2: $a \in T_{n-1}$. If $b \in T_{i j}$, it follows from (SE1) that $[a, b]=e$. If $b \in T_{i}$, it follows from (E2) that $[a, b] \in T_{i,-(n-1)}$.

Case 3: $a \in T_{n-1,-n}$. Assume that $b \in T_{i j}$. If $j<0$, it follows from (S3) that $[a, b]=e$. If $j=n-1$, it follows from (S4) that $[a, b] \in T_{i,-n}$. If $j=n$, it follows from (S4) that $[a, b] \in T_{i,-(n-1)}$. Assume that $b \in T_{i}$. It follows from (SE1) that $[a, b]=e$. Hence $a b=b^{\prime} a$ for some $b^{\prime} \in B$ and $U^{+}=\langle B\rangle\langle A\rangle=\langle B\rangle Y^{+}$.

Let $\alpha=\beta \gamma \in U^{+}$, where $\beta \in\langle B\rangle$ and $\gamma \in Y^{+}$. Since $\sigma \beta \sigma^{-1}$ lies in $U^{+}$for any $\sigma \in Y^{-}$, we have $\sigma \alpha=\sigma \beta \sigma^{-1} \cdot \sigma \gamma \in U^{+} Y^{-} Y^{+}$.

(ii) Similar proof as (i).

(iii) Follows from (i) and (ii).

Set $S:=\left\{\alpha \in L_{n} \mid \alpha_{n-1,1}=\alpha_{n 1}=0\right\}$.

Lemma 4.3. Let $n \geq \operatorname{sr}(R)+2$. Then, for every $\alpha \in L_{n}$, there exist $\beta_{\alpha} \in V^{+}$and $\gamma_{\alpha} \in V^{-}$such that $\gamma_{\alpha} \beta_{\alpha} \alpha \in S$.

Proof. Let $\alpha \in L_{n}$, and let $v$ be the first column of $\alpha$. It follows from Lemma 3.4 that there exists a matrix $B \in \mathrm{M}_{(n-2) \times 2}(R)$ such that the first $n-2$ coordinates of

$$
v^{\prime}=\Phi\left(\left(\begin{array}{cc}
E_{n-2} & B \\
0 & E_{2}
\end{array}\right)\right) v
$$

form a unimodular column. Now, there exists another matrix $C \in \mathrm{M}_{2 \times(n-2)}(R)$ such that both $(n-1)$-th and $n$-th coordinates of

$$
\Phi\left(\left(\begin{array}{cc}
E_{n-2} & 0 \\
C & E_{2}
\end{array}\right)\right) v^{\prime}
$$


are 0 . Let

$$
\beta_{\alpha}=\Phi\left(\left(\begin{array}{cc}
E_{n-2} & B \\
0 & E_{2}
\end{array}\right)\right) \in V^{+}, \quad \gamma_{\alpha}=\Phi\left(\left(\begin{array}{cc}
E_{n-2} & 0 \\
C & E_{2}
\end{array}\right)\right) \in V^{-}
$$

Then we have $\gamma_{\alpha} \beta_{\alpha} \alpha \in S$.

Corollary 4.4. Let $n \geq \operatorname{sr}(R)+2$. Then $U_{n} U_{n}^{-} L_{n} \subseteq U^{+} U^{-} S$.

Proof. Let $\alpha \in L_{n}$. Since $\beta_{\alpha}$ normalizes $U_{n}^{-}$, it follows from Lemma 4.3 that

$$
U_{n} U_{n}^{-} \alpha=\left(U_{n} \beta_{\alpha}^{-1}\right)\left(\beta_{\alpha} U_{n}^{-} \beta_{\alpha}^{-1} \gamma_{\alpha}^{-1}\right)\left(\gamma_{\alpha} \beta_{\alpha} \alpha\right) \in U^{+} U^{-} S .
$$

Lemma 4.5. Let $n \geq \operatorname{sr}(R)+$ 2. Then $Y^{-} U^{+} U^{-} S \subseteq U^{+} U^{-} L_{n} F_{n}$.

Proof. Let $\alpha \in S$ and $\beta \in\left(Y^{-}\right)^{\alpha}$. Since $\left(Y^{-}\right)^{\alpha} \subseteq U_{n}^{-}$, we have

$$
\beta=\left(\begin{array}{ccc}
E_{n} & 0 & 0 \\
A & 1 & 0 \\
C & B & E_{n}
\end{array}\right)
$$

for some $A \in \mathrm{M}_{1 \times n}(R), B \in \mathrm{M}_{n \times 1}(R)$ and $C \in \mathrm{M}_{n \times n}(R)$. By the definition of $S$, the first column of $\alpha$ remains unchanged if we multiply $\alpha$ on the left by an element of $Y^{-}$. Hence the first column of $\beta$ coincides with that of the identity matrix. Then $A_{11}=0$ and $C_{* 1}=0$. It follows from Lemma 3.3 that $C_{n *}=0$ and $B_{n 1}=0$. Therefore, we have $\beta \in U_{n}^{-} \cap F_{n} \subseteq F_{n}$. Since $\left(Y^{+}\right)^{\alpha} \subseteq F_{n}$, by Lemma 4.2, we have

$$
\begin{aligned}
& Y^{-} U^{+} U^{-} \alpha \subseteq U^{+} U^{-} Y^{+} Y^{-} \alpha \\
& \quad=U^{+} U^{-} \alpha\left(Y^{+}\right)^{\alpha}\left(Y^{-}\right)^{\alpha} \subseteq U^{+} U^{-} L_{n} F_{n} .
\end{aligned}
$$

Lemma 4.6. Let $\sigma \in U_{2 n+1}(R, \Delta)$ have the form

$$
\left(\begin{array}{ccc}
E_{n} & 0 & 0 \\
A & 1 & 0 \\
C & B & E_{n}
\end{array}\right)
$$

where $A \in \mathrm{M}_{1 \times n}(R), B \in \mathrm{M}_{n \times 1}(R), C \in \mathrm{M}_{n \times n}(R)$. Then $\sigma \in E U_{2 n+1}(R, \Delta)$. Proof. $\sigma^{-1}$ has the form

$$
\left(\begin{array}{ccc}
E_{n} & 0 & 0 \\
-A & 1 & 0 \\
B A-C & -B & E_{n}
\end{array}\right)
$$


Let $i<0<j$. It follows from Lemma 3.3 that

$$
B_{n-j+1,1}=-\bar{A}_{1 j} \mu, \quad B_{n+i+1,1} A_{1 j}-C_{n+i+1, j}=\bar{C}_{n-j+1,-i} \lambda
$$

and $\left(A_{1 j}, C_{n-j+1, j}\right) \equiv(0,0) \bmod \Delta$. Then $\left(A_{1 j}, C_{n-j+1, j}\right) \in \Delta$ and

$$
\sigma=\prod_{j=1}^{n} T_{j}\left(A_{1 j}, C_{n-j+1, j}\right) \prod_{j-i<n+1} T_{i j}\left(C_{n-j+1,-i}\right) .
$$

Hence $\sigma \in E U_{2 n+1}(R, \Delta)$.

Now we have the following decomposition theorem.

Theorem 4.7 (Decomposition theorem). Let $n \geq \operatorname{sr}(R)+2$. Then every element of $E U_{2 n+1}(R, \Delta)$ has a $G_{n} U_{n}^{-} F_{n}$-decomposition, that is,

$$
E U_{2 n+1}(R, \Delta)=G_{n} U_{n}^{-} F_{n} .
$$

Proof. It suffices to prove that $G_{n} U_{n}^{-} F_{n}$ is stable under left multiplications by the generators of $E U_{2 n+1}(R, \Delta)$. By Lemma 4.1, it is enough to show that

$$
Y^{-} G_{n} U_{n}^{-} F_{n} \subseteq G_{n} U_{n}^{-} F_{n} .
$$

Since $L_{n}$ normalizes $U_{n}$ and $U_{n}^{-}$, we have

$$
G_{n} U_{n}^{-} F_{n}=U_{n} L_{n} U_{n}^{-} F_{n}=U_{n} U_{n}^{-} L_{n} F_{n} .
$$

It follows from Corollary 4.4 and Lemma 4.5 that

$$
\begin{aligned}
Y^{-} G_{n} U_{n}^{-} F_{n} & =Y^{-} U_{n} U_{n}^{-} L_{n} F_{n} \\
& \subseteq Y^{-} U^{+} U^{-} S F_{n} \subseteq U^{+} U^{-} L_{n} F_{n} \subseteq G_{n} U_{n}^{-} F_{n} .
\end{aligned}
$$

Proof of Theorem 1.1. Let

$$
\sigma \in U_{2(n-1)+1}(R, \Delta) \cap E U_{2 n+1}(R, \Delta) .
$$

By Theorem 4.7, we have $\sigma=\alpha \beta \gamma$, where

$$
\alpha=\left(\begin{array}{ccc}
A^{11} & A^{12} & A^{13} \\
0 & 1 & A^{23} \\
0 & 0 & A^{33}
\end{array}\right) \in G_{n}, \quad \beta=\left(\begin{array}{ccc}
E_{n} & 0 & 0 \\
B^{21} & 1 & 0 \\
B^{31} & B^{32} & E_{n}
\end{array}\right) \in U_{n}^{-}, \quad \gamma \in F_{n} .
$$


Since the first columns of both $\sigma$ and $\gamma$ coincide with that of the identity matrix, we get $A^{33}\left(B^{31}\right)_{* 1}=0$ and $B_{11}^{21}+A^{23}\left(B^{31}\right)_{* 1}=0$. Since $A^{33}$ is invertible, we have $\left(B^{31}\right)_{* 1}=0$ and $B_{11}^{21}=0$. Hence $\beta \in F_{n}$. Thus we can assume that $\sigma=\alpha \gamma$. Since the first columns of both $\sigma$ and $\gamma$ coincide with that of the identity matrix, we get that $\left(A^{11}\right)_{* 1}$ equals the first column of the identity matrix and $\left(A^{33}\right)_{n *}$ equals the last row of the identity matrix. Hence we can express $\alpha$ as $\alpha=\alpha_{1} \alpha_{2}$, where $\alpha_{1} \in U_{2(n-1)+1}(R, \Delta)$ and $\alpha_{2} \in F_{n}$. Thus we can assume that $\sigma=\alpha \gamma$ with $\alpha, \gamma \in U_{2(n-1)+1}(R, \Delta)$.

Since

$$
\alpha=\left(\begin{array}{ccc}
E_{n} & A^{12} & A^{13}\left(A^{33}\right)^{-1} \\
0 & 1 & A^{23}\left(A^{33}\right)^{-1} \\
0 & 0 & E_{n}
\end{array}\right)\left(\begin{array}{ccc}
A^{11} & 0 & 0 \\
0 & 1 & 0 \\
0 & 0 & A^{33}
\end{array}\right),
$$

where the second matrix lies in $L_{n}$, we get that $A^{11} \in E_{n}(R)$. Let $A^{11}=\left(\begin{array}{cc}1 & 0 \\ 0 & A_{*}^{11}\end{array}\right)$, we have $A_{*}^{11} \in E_{n}(R) \cap \mathrm{GL}_{n-1}(R)$. Since $n \geq \operatorname{sr}(R)+2$, it follows from the stability of the $K_{1}$-functor under the stable range condition $S_{n}$ [6, Theorem 5.4.2] that $A_{*}^{11} \in E_{n-1}(R)$. Then $\alpha \in E U_{2(n-1)+1}(R, \Delta)$.

Since $\gamma \in F_{n}$, we can express $\gamma$ as

$$
\gamma=\left(\begin{array}{ccc}
1 & 0 & 0 \\
0 & C & 0 \\
0 & 0 & 1
\end{array}\right)\left(\begin{array}{ccc}
1 & C^{12} & C^{13} \\
0 & E_{2(n-1)+1} & C^{23} \\
0 & 0 & 1
\end{array}\right)
$$

where $C \in E U_{2(n-1)+1}(R, \Delta)$. It follows from $\gamma \in U_{2(n-1)+1}(R, \Delta)$ that

$$
C^{12}=0, \quad C^{13}=0 \quad \text { and } \quad C^{23}=0 .
$$

Then $\gamma \in E U_{2(n-1)+1}(R, \Delta)$. Hence $\sigma \in E U_{2(n-1)+1}(R, \Delta)$.

Acknowledgments. The author is grateful to the referee for constructive suggestions for improvements. The author would like to thank Prof. Guoping Tang for helpful discussions.

\section{Bibliography}

[1] A. A. Ambily, Normality and $K_{1}$-stability of Roy's elementary orthogonal group, J. Algebra 424 (2015), 522-539.

[2] A. Bak and G. Tang, Stability for Hermitian $K_{1}$, J. Pure Appl. Algebra 150 (2000), no. 2, 109-121.

[3] A. Bak, V. Petrov and G. Tang, Stability for quadratic $K_{1}, J$. K-Theory 30 (2003), $1-11$. 
[4] A. Bak and R. Preusser, The E-normal structure of odd dimensional unitary groups, J. Pure Appl. Algebra 222 (2018), no. 9, 2823-2880.

[5] H. Bass, K-theory and stable algebra, Inst. Hautes Études Sci. Publ. Math. (1964), no. 22, 5-60.

[6] H. Bass, Algebraic K-theory, W. A. Benjamin, New York, 1968.

[7] V. A. Petrov, Odd unitary groups, J. Math. Sci. 130 (2005), 4752-4766.

[8] S. Sinchuk, Injective stability for unitary $K_{1}$, revisited, J. K-Theory 11 (2013), no. 2 , 233-242.

[9] G. P. Tang, Unitary $K_{1}$-groups under $\Lambda$-stable range condition (in Chinese), Chinese Ann. Math. Ser. A 25 (2004), no. 2, 171-178; translation in Chinese J. Contemp. Math. 25 (2004), 143-152.

[10] L. N. Vaseršteĭn, On the stabilization of the general linear group over a ring, Math. USSR-Sb. 8 (1969), 383-400.

[11] W. Yu, Stability for odd unitary $K_{1}$ under the $\Lambda$-stable range condition, J. Pure Appl. Algebra 217 (2013), no. 5, 886-891.

[12] W. Yu and G. Tang, Nilpotency of odd unitary $K_{1}$-functor, Comm. Algebra 44 (2016), no. 8, 3422-3453.

Received July 4, 2019; revised October 13, 2019.

\section{Author information}

Weibo Yu, School of Sciences, Chang'an University,

Xi'an, 710064, P. R. China.

E-mail: yuwb@chd.edu.cn 